von Muralt, J: Exercitationes medicae seu Experimenta Anatomica. Zürich 1675.

- - Vademecum anatomicum sive clavis medicinae. Zürich 1677.

- - Anatomisches Collegium. Zürich 1687.

Nabholz, H.: Zürichs Höhere Schulen von der Reformation bis zur Gründung d. Universität, 1525-1833. In Festschr. zur Jahrhundertfeier d. Zürcherischen Schulen. Bd. III, 1938.

Neuburger, M. u. J. Pagel: Handbuch d. Gesch. d. Medizin. Bd. I-III, 1902/1905.

Obschlager, O.: Der Zürcher Stadtarzt J. v. Muralt u. d. medizinische Aberglaube seiner Zeit. Inaug. Diss. Zch. 1926.

Peters, H.: Der Arzt u. d. Heilkunst in d. deutschen Vergangenheit. Lpz. 1900.

Römer, J. J.: Conrad Geßner. 21. Neujahrsblatt d. Naturforsch. Ges. 1819.

Roth, M: Andreas Vesailius Bruxellensis. Berlin 1892.

Sprengel, K.: Versuch einer pragmatischen Geschichte der Arzneikunde. Bd. III u. IV.

Töply, Ritter von: Geschichte d. Anatomie. In Handbuch d. Gesch. d. Medizin (Neubur. ger u. Pagel) Bd. II. 1903.

Vesalius, A.: De humani corporis fabrica libri septem. Basel 1543.

Wehrli, G. A.: Der Zürcher Stadtarzt Dr. Chr. Clauser u. seine Stellung zur Reformation der Heilkunde im 16. Jahrhundert. 1923.

- - Die Bader, Barbiere u. Wundärzte im alten Zürich. Mitt. d. Antiquar. Ges. Zch. 30, H. 3, 1927.

-- - Die Wundärzte u. Bader Zürichs als zünftige Organisation. (Geschichte d. Gesellschaft z. Schwarzen Garten). Ebenda 30, H. 8, 1931.

- - Die Krankenanstalten u. d. öffentlich angestellten Ärzte und Wundärzte im alten Zürich. Ebenda 31, H. 3, 1934.

$E^{\prime}$ olf, R.: Biographien zur Kulturgeschichte d. Schweiz. Bd. I-III, 1858/60.

Otto Bucher.

\title{
Note sur deux documents linnéens conservés à Genève
}

Les collections du Conservatoire botanique de Genève renferment deux Rarissima qui sont liés très étroitement au nom de Charles Linné (1707 à 1778), l'éminent naturaliste suédois. Dans l'un de ces cas, il s'agit d'une plante récoltée par ce savant lui-même; dans l'autre, d'une des publications les plus rares qu'on connaisse de Linné.

I.

La plante en question - représentée par deux petites tiges fleuries, parfaitement conservées - est le Diapensia lapponica. C'est une espèce suffrutescente de la toundra, formant de denses gazons, comme nos Loiseleuria ou Empetrum; ses fleurs, solitaires et longuement pédonculées, sont d'un beau blanc laiteux. Son aire comprend la zone circumpolaire subarctique à arctique. Dans le système végétal, le genre Diapensia constitue, avec cinq autres genres et une totalité d'environ douze 
espèces, la famille des Diapensiacées, voisine des Ericacées. Les deux spécimens ont été récoltés par Linné (qui avait alors 25 ans), en 1732, lors de son voyage fait en Laponie, et donnés au Conservatoire botanique de Genève deux cents ans plus tard, en 1933, par un Français, le Commandant Alfred Saint-Yves, que des liens d'amitié attachaient à Genève.

Mais comment la plante était-elle arrivée dans les mains de Saint-Yves? C'est là toute une odyssée, et s'il est exact que les livres ont leur histoire, et même leur destin - habent sua fata libelli -, il n'est pas moins viai que les plantes d'herbier ont souvent leurs aventures. Tel est le cas de notre Diapensia.

Linné mourut en 1778. A la mort de son fils, qui s'appelait aussi Charles, en 1783, les collections importantes de Linné furent acquises par un Anglais, Sir James Edward Smith, pour le prix de 1000 guinées, et transportées d'Upsal à Londres. En 1789, Smith, voulant faire plaisir à un botaniste ami, d'origine écossaise, établi à Orbe et devenu vaudois, Edmond Davall (1763-1798), sortit de l'herbier nos deux échantillons pour les envoyer en Suisse avec une charmante dédicace. Grandes furent la joie et la reconnaissance du destinataire: dans ses lettres adressées à Smith $^{1}$ - où il raconte entre autres ses herborisations faites dans le Jura vaudois - Davall ne parle pas moins de trois fois de «this inestimable present» et dit qu'il n'entre jamais dans sa chambre de travail sans faire une révérence vers le Diapensia lapponica, mis sous verre et placé, avec les livres de Linné, au «coin sacré» de sa bibliothèque. Ce précieux cadeau resta pendant plus de cent ans dans la famille Davall: à la mort d'Edmond Davall, il fut transmis à son fils Edmond (1793-1860), inspecteur forestier de l'Etat de Vaud et colonel, puis à son petit-fils Albert (1821-1892), inspecteur forestier et botaniste. En 1892, Albert Davall légua le Diapensia à son ami Emile Burnat (1828-1920), l'explorateur de la flore des Alpes-Maritimes et de la Corse, qui lui donna une place d'honneur dans la bibliothèque de son magnifique herbier à Nant sur Vevey. En 1923, le fils de ce dernier, Jean Burnat (1872-1939), ampélographe et viticulteur, en fit cadeau à un intime d'Emile Burnat, le Commandant Alfred Saint-Yves (1855-1933), collaborateur à la Flore des Alpes-Maritimes de Burnat et auteur de travaux sur les Graminées. Ainsi le Diapensia s'en alla en France, à Vernou-sur-Brenne, dans le département d'Indre-et-Loire, où il resta jusqu'à la mort de Saint-Yves. Selon

1 Cf. Lady Smith: Memoir and correspondence of the late Sir James Edward Smith. Londres 1832. Vol. II. 
un vœu du défunt, il fut légué, avec une partie de son herbier, au Conservatoire botanique de Genève, en souvenir de John Briquet (1870-1931), ancien directeur de cette institution et ami personnel de Saint-Yves, et revint ainsi en Suisse.

Le voyage en Laponie au cours duquel Linné a récolté de Diapensia, a duré de mai à novembre 1732. Le jeune explorateur remonta la Suède vers le nord, jusqu'au fond du golfe de Bothnie, traversa la Laponie et gagna le cap Nord. Ce long trajet, entrepris au nom de la Société des Sciences d'Upsal et après recommendation de l'astronome André Celsius, fut pénible et plein de dangers. Linné, à pied et seul, n'emporta que son journal, deux chemises et les habits qu'il avait sur lui, une demi-toise pour prendre des mesures, et un petit porte-feuille renfermant du papier et des plumes. Une peau lui servait à la fois de matelas et de manteau. Dans les montagnes, il retrouva l'hiver: le sol humide et marécageux était couvert de neige et de glaces, les rivières impraticables. Puis il atteignit des régions où, ne comprenant plus la langue du pays, il était à la merci des indigènes auxquels il ne pouvait demander même de quoi se nourrir. Toujours errant au milieu de tristes forêts, entouré de gens grossiers dont il n'était pas possible d'obtenir le moindre renseignement utile, n'ayant ni pain, ni sel, ni boissons fermentées, il vécut presque exclusivement de petits poissons desséchés.

Les résultats botaniques de ce long voyage - plus de 1000 milles faits à pied - sont contenus dans un beau volume de 400 pages: Flora Lapponica, publié par Linné cinq ans plus tard (Amsterdam 1737). Cette flore renferme (p.55-56) une description du Diapensia ${ }^{2}$ et, sur la planche I, dédiée au professeur Jean Burman, à Amsterdam, une excellente figure de la plante en grandeur naturelle. C'est seulement dans un ouvrage postérieur de Linné, le Species plantarum (1753), que le binôme Diapensia lapponica apparaît (p.141); on y trouve encore un second Diapensia: le D. helvetica, que les botanistes ont cependant séparé plus tard du genre Diapensia et qui est devenu l'Androsace helvetica, plante bien connue et croissant dans nos hautes Alpes calcaires.

Ajoutons que Diapensia était chez les anciens le nom d'une plante officinale toute différente, le sanicle (Sanicula europaea) — c'est encore aujourd'hui un des noms italiens de cette espèce - et Linné l'a trans-

2 Sous le nom de «Diapensia», avec citation des synonymes de Tournefort, Bauhin, Clusius et Parkinson. 
féré, sans raison sérieuse, simplement parce qu'il était disponible, à la plante nordique.

II.

En 1921, lors de l'acquisition de la bibliothèque de Candolle, le Conservatoire botanique de Genève est entré en possession d'un ouvrage de Linné extrêmement rare, intitulé: Orbis eruditi judicium de Caroli Linnaei scriptis.

C'est une brochure de 16 pages petit in- $8^{03}$ parue à Stockholm en 1741. Elle est sans pagination. Lieu et date ne sont pas indiqués; l'auteur - qui était Linné lui-même - non plus. La brochure n'a pas été mise en vente mais a été distribuée par Linné à ses amis les plus intimes seulement. C'est le seul ouvrage que Linné ait publié à titre anonyme.

Cette brochure est considérée comme très rare par tous les bibliographes. On ignore le nombre exact des exemplaires connus actuellement. Selon Junk, libraire berlinois, qui en a publié une édition facsimilaire en 1901, il n'en existerait que quatre. ${ }^{4}$ Cependant, le botaniste hongrois Flatt, auquel on doit une étude précieuse sur cet opuscule, ${ }^{5}$ faisait remarquer que le nombre des exemplaires est probablement un peu plus élevé. Pritzel ${ }^{6}$ ne vit la brochure qu'à la bibliothèque de Candolle, à Genève - c'est l'exemplaire que nous avons en mains et qui est relié, avec 16 autres brochures, au volume 1 d'une série d'Antiqua portant le nom: Opuscules botaniques. A la fameuse bibliothèque de Kew (Angleterre), l'ouvrage manque. Par contre, le Musée Britannique, à Londres, en possède ${ }^{7}$ un exemplaire provenant de la bibliothèque de Linné et donné, en 1907, par la Société Linnéenne de Londres.

3 Certains auteurs, et récemment le «Catalogue of the works of Linnaeus preserved in the libraries of the British Museum», 2e édition (Londres 1933), p. 163, indiquent, par erreur, comme format: in-12.

4 W. Junk: Bibliographia Linnaeana. Berlin 1902, p.6. (《Gegenüber diesem Rarissimum verschwinden in bezug auf Seltenheit die vielen anderen gesuchten Schriften Linné's, selbst die editio princeps des ,Systema Naturae'. Es ist eine der seltensten Flug. schriften überhaupt.»)

5 K. A. Flatt: Linnének névtelenul Riadott apologiája. Die anonym verfaßte Apologie I.inné's. Magyar Bot, Lapok 1, p. 76-84, 1902. — Cf. aussi Flatt in: Bot. Centralbl. 66, p. 217, 1896.

6 G. A. Pritzel: Thesaurus literaturae botanicae. Leipzig 1851, p. 168. 2. édition, 1872, p. 187.

7 Cf. note 3 . 
Dès son apparition, cette brochure était si rare qu'en 1792 déjà l'Allemand Stœver l'a rééditée dans son ouvrage intitulé: Collectio epistolarum quas ad viros illustres et clarissimos scripsit Carolus a Linné. Accedunt opuscula pro et contra virum immortalem scripta, extra Sueciam rarissima. ${ }^{8}$ La même année, Stœver, dans sa biographie de Linné, en donnait un extrait en langue allemande. ${ }^{9}$ Les trois premières pages de celui-ci ont été copiées plus tard par Pritzel. ${ }^{10}$

L'opuscule en question est une apologie écrite par Linné contre ses adversaires dont le plus redouté était le minéralogiste J. G. Wallerius. ${ }^{11}$ Après une absence de plusieurs années, passées à l'étranger, Linné était rentré, en 1738, à Stockholm, où il avait été reçu avec froideur. En 1741, lorsque la chaire de Lars Roberg, professeur de médecine et d'anatomie à Upsal, devint vacante, les adversaires de Linné tentèrent de l'empêcher de succéder à celui-ci. Alors, pour rendre ses compatriotes attentifs à ses distinctions et à ses mérites, Linné publia cette brochure, qui contient: $1^{0}$ une liste des dates principales de la vie de Linné; $2^{0}$ l'énumération de ses ouvrages, publiés de 1735 à $1740 ; 3^{0}$ le jugement des auteurs contemporains sur ces mêmes ouvrages.

Ces témoignages ont été choisis dans les lettres et publications de vingt étrangers. Tous jouissaient de quelque gloire dans le monde savant. On y trouve: cinq Hollandais: van Gorter, Boerhaave, van Royen, Gronovius et Jean Burman, le célèbre explorateur de la flore de Ceylan; quatre Anglais: Sloane, connu par son ouvrage sur la flore de la Jamaïque, alors président de l'Académie Royale à Londres; Dillenius, professeur à Oxford, auteur du fameux «Hortus Elthamensis»; les médecins Isaac Lawson et Donell Jacob; quatre Français: de Sauvages, Antoine de Jussieu, Barrère et Gravel; deux Suisses: Jean Gessner (1709-1790), professeur de mathématiques et de physique à Zurich, et Albert de Haller (1708-1777), alors professeur à Goettingue; enfin cinq Allemands: Gleditsch, professeur à Berlin; J.Ph. Breyne, médecin à Dantzig; les professeurs Lange et Kohl; le conseiller aulique Menken.

Citons ici deux de ces témoignages. Notre compatriote, A. de Haller, dit (Acta Germ. Eruditorum p. 288): "Wir machen uns eine Freude, gleich im Anfange zu sagen, daß noch kein Buch in dieser Wissenschaft geschrieben sei, welches man [mit] Linnaei Genera plantarum einiger-

8 Hambourg 1792; notre opuscule s'y trouve aux p. 159-172.

9 D. H. Stœver: Leben des Ritters Carl von Linné. Hambourg. Vol. 1, 1792, p. 240_253.

10 Pritzel 11. cc.

11 Wallerius publia, en février 1741, une brochure contre Linné. 
maßen vergleichen könnte. Es ist der ganze Riss davon unentlehnt, unversucht und neu, die Ausarbeitung aus einer scharfen Untersuchung von acht tausend Pflanzen genommen... Aber was Linnaeus getan, hat niemand versucht, noch getan.» Et le Hollandais H. Bœrhaave, professeur de botanique à Leyde, écrit dans une lettre adressée à Linné le 13 janvier 1737, à propos du même ouvrage: «Secula laudabunt, boni imitabuntur, omnibus proderit.»

A la fin de la brochure, on trouve quelques critiques anonymes, tirées de revues scientifiques, ainsi qu'une liste bibliographique renvoyant le lecteur à d'autres témoignages sur les ouvrages de Linné.

Mais revenons à la liste biographique contenue au début de la brochure. Elle commence comme suit: «1707 Maji 13 natus». C'est là, comme le fait déjà remarquer Flatt, ${ }^{12}$ l'indication la plus authentique de la date de naissance de Linné! Linné suivait le calendrier julien. A cette époque (jusqu'en 1800), la différence entre les calendriers julien et grégorien était de 11 jours. Linné, donc, d'après le nouveau calendrier, est né le 24 mai 1707 - et non pas le 23 mai comme l'indiquent la plupart des auteurs.

Il ne nous semblait pas inutile d'attirer l'attention des naturalistes sur ce fait, déjà constaté par Flatt (l. c.), dont l'article paraît être resté peu connu.

Ajoutons que l'attaque de Wallerius, qui a provoqué en première ligne cette publication rarissime, n'eut pas de succès: le 5 mai 1741 , Linné fut nommé professeur à l'académie d'Upsal et succéda ainsi à Roberg.

A. Becherer.

\section{Eine Vorlesung über die Symphysentrennung von Rudolf Abraham Sdhiferli (1775-1837)*}

Auf die Idee, durch Trennung der beiden Schambeine in der Symphyse unter der Geburt eine gewisse Erweiterung des weiblichen Beckens zu

12 Flatt 1. c. 1902 , p. 83.

* Biographische Notiz: Rudolf Abraham Schiferli wurde am 30. September 1775 in Thun geboren und starb am 3. Juni 1837 in Bern. Er studierte in Bern und Jena, wo er 1795 zum Doktor der Medizin und Chirurgie promovierte. Zur weiteren Ausbildung ging er nach Paris, wo er unter Boyer und Larrey, dann nach Wien, wo er unter Johann Peter Frank arbeitete. In seine Heimat zuruckgekehrt, erwarb er sich bald den Ruf eines aus- 